\title{
Innovative Use of Medical Infrared Thermography to Evaluate Adhesive Properties of Two Buprenorphine Transdermal Patch Formulations in a Single Dose Adhesion Performance Study
}

\author{
Duna SN ${ }^{1 *}$, Ghita $\mathbf{A}^{1}$, Grasser $\mathbf{S}^{2}$, Rizea- Savu $\mathbf{S}^{3}$ \\ ${ }^{1} 3 S$ Pharmacological Consultation \& Res. SRL, Romania \\ ${ }^{2} \mathrm{AMW} \mathrm{GmbH}$, Warngau, Germany \\ ${ }^{3} 3 \mathrm{~S}-$ Pharmacological Consultation \& Research $\mathrm{GmbH}$, Harpstedt, Germany
}

\begin{abstract}
An innovative approach for the assessment of transdermal patches adherence to the skin was developed and applied post-hoc to supportive imaging data collected in an open label, two-period, two-sequence, two-way crossover, controlled, randomized, single dose study to assess adhesion performance of two buprenorphine formulations (generic versus originator), applied topically to healthy male and female volunteers. The technology used for data acquisition is called Medical Infrared Thermography (MIT), a non-invasive, non-radiating imaging technique for surface temperature mapping. The use of this technology in an adhesion performance study was based on the rationale that the surface of a transdermal patch reaches a temperature in equilibrium with that of the body area where it is applied and whenever a discontinuity between skin and patch exists, the transmission of thermic energy to the surface of the transdermal patch changes. In case air is interposed, the transmission is minimal so any cases of patch lift-off from skin become visible on thermograms. The thermograms were acquired in standardized conditions but were initially intended only as supportive imaging technique, while the primary adhesion performance data was acquired through the standard approach of visual examination coupled with manual markings. In this article we further demonstrate the value of the acquired thermograms as main adhesion assessment tools, used in conjuncture with an in-house validated image processing software for the actual quantification of percentages of detachment. This novel approach for automated and unbiased analysis of adhesion performance was shown to be highly reliable and reproducible, attributes that recommend it for future use as primary adhesion assessment tool pending completion of full method validation.
\end{abstract}

Keywords: Adhesion performance study; Bioequivalence; Transdermal patches; Medical infrared Thermography; Innovative adhesion assessment tools

\section{Introduction}

Transdermal patches are dosage forms used to deliver a therapeutically effective amount of drug across a patient's skin. One of the critical factors affecting the performance of transdermal patches is skin adhesion. Poorly adhered patches can result in low drug permeation leading to a reduced efficacy or can even pose a safety risk in case of accidental transfer from intended patient to another person. On the other hand, patches that adhere too strongly can cause skin tearing, bleeding or inflammation at removal. Therefore, evaluation of adhesion performance is a very important step in the development program of new transdermal patches as well as generics. Current methodology for adhesion evaluation requires the development of grid patterns of the same shape and area like the evaluated transdermal patches, which are pre-printed on transparent plastic sheets [1]. The assessor identifies areas of patch detachment visually and then marks them directly on the grid pattern that he is holding superposed to the patch. While the drawing is made, the grid must be kept in close vicinity of the patch, directly above it, but the assessor has to avoid contact between the two as that might cause re-attachment of the patch to the skin and bias subsequent assessments. The grids are then used for quantification of overall detached area. The evaluation is typically done by a human evaluator counting the boxes of the grid marked, a method that is quite dated and not easily reproductible (because boxes are very seldom either fully marked or fully unmarked and the fractions marked are hard to quantify, leading typically to high variability between assessors). In order to avoid bias due to the partlymarked boxes, a commonly accepted approach is to consider any box that contains some black as fully marked. The downside is that an overestimation of detached area occurs, the data becoming quite crude for the discriminative statistical analysis currently required by EMA Guidelines (90\% confidence interval approach, applied to adhesion percentages as continuous variables) [2].

The more modern approach requires the use of image processing software for the counting of pixels marked and conversion of pixels to detachment area. In the present study, the latter technique was employed for quantification of adhesion percentages from marked grids and the data collected was considered for comparative assessment of adhesion performance between the Test and Reference patch.

While unanimously accepted as standard methodology, the visual evaluation of adhesion performance does have some noteworthy disadvantages: marking the areas of detachment manually can prove to be a time-consuming activity; it is many times very difficult to make a visual distinction between real detachment (defined as no contact between transdermal patch and skin) and an irregular appearance of

*Corresponding author: Duna SN, Research Project Manager, 3S Pharmacological Consultation \& Res SRL, Romania, Tel: +40 758109 202; E-mail: nicoleta. duna@3spharma.eu

Received November 21, 2018; Accepted December 12, 2018; Published December 19, 2018

Citation: Duna SN, Ghita A, Grasser S, Rizea- Savu S (2018) Innovative Use of Medical Infrared Thermography to Evaluate Adhesive Properties of Two Buprenorphine Transdermal Patch Formulations in a Single Dose Adhesion Performance Study. J Bioequiv Availab 10: 92-98. 384. doi: 10.4172/0975-0851.1000384

Copyright: $\odot 2018$ Duna SN, et al. This is an open-access article distributed under the terms of the Creative Commons Attribution License, which permits unrestricted use, distribution, and reproduction in any medium, provided the original author and source are credited. 
Citation: Duna SN, Ghita A, Grasser S, Rizea- Savu S (2018) Innovative Use of Medical Infrared Thermography to Evaluate Adhesive Properties of Two Buprenorphine Transdermal Patch Formulations in a Single Dose Adhesion Performance Study. J Bioequiv Availab 10: 92-98. 384. doi: 10.4172/0975-0851.1000384

a patch which can be caused by an uneven skin surface or the often observed phenomenon of skin and patch wrinkling together while in full contact (typical for formulations worn for several days); there is a hypothetical risk of unwillingly biasing the data by pressing the grid against the patch while performing the markings or by overlooking to mark some of the more tiny areas of detachment. The visual method can also suffer bias through subjective evaluation and evaluator-toevaluator variability, being thus very difficult to standardize. For the comparative evaluation of adhesion performance required for generics, FDA Guidance recommends blinding of the Test and Reference products, whenever possible, in order to avoid subjective evaluation as a source of bias [3]. However, it is also acknowledged that blinding may not be possible in instances where the appearance of a transdermal patch reveals its identity (as it is usually the case for the Reference). At the same time, the use of overlays or covers for the purpose of blinding is not justified considering the risk of affecting the performance of the products under investigation.

The purpose of our research was to overcome the disadvantages of current approaches by developing an unbiased, automated system for adhesion data acquisition and processing, based on modern-days technology. Medical Infrared Thermography (MIT) is a non-invasive, non-radiating imaging technique used for surface temperature mapping [4]. Well-established applications of thermography range from industrial material testing [5] to diagnostic biomedical applications in circulatory and/or inflammatory disorders that have impact on the thermal homeostasis of certain body regions (such as inflammatory rheumatoid arthritis, diabetic neuropathy, Raynaud syndrome, nasal congestion, knee inflammation etc.) [6]. In the particular case of our proposed MIT application it has been considered that the surface of a transdermal patch will reach a temperature in equilibrium with that of the body area where it is applied. In any situation in which a discontinuity between skin and patch exists, the transmission of thermic energy to the surface of the transdermal patch will change depending on the thermal transmission properties of the fluid filling the space. In case air is interposed the transmission will be minimal (heat transfer coefficient of air distributed without convection ranges from 5 to $37 \mathrm{~W} / \mathrm{m}^{2} \mathrm{~K}$ [7]) while in case of a liquid it will be very similar to the interface normally present between the skin and the patch (heat transfer coefficient of water and liquids distributed without convection ranges from 50 to $3000 \mathrm{~W} / \mathrm{m}^{2} \mathrm{~K}$ [7]). It is noteworthy that in practice the situation involving an air gap is the one in which the drug cannot be transported from the patch itself to the skin, so all areas exhibiting this type of detachment represent losses in the drug transportation process and their determination is important in the characterization of adhesion performance. In case the gap has liquid filling (liquid exudation from skin and/or the matrix of the patch), the continuity of the absorption process is maintained and therefore these areas are not critical like the ones filled with air.

A Meditherm med $2000^{m}$ thermographic system for biomedical applications was used for patch surface temperature mapping in the present study. The camera is designed and calibrated for optimal operation in the narrow temperature range specific for recording metabolic heat $\left(10^{\circ} \mathrm{C}-40^{\circ} \mathrm{C}\right)$, with a thermal resolution of $0.1^{\circ} \mathrm{C}$. Extremely accurate readings were obtained as each pixel from the output represents an individual referenced measurement.

The use of ambient temperature forced airflow, oriented towards the patch surface, was employed in order to enhance the temperature gradient between attached and detached areas of the patch for optimal resolution.

\section{Materials and Methods}

\section{Investigational products}

The Reference product, Transtec $\mathrm{PRO}^{\circledast} 70$ Mikrogramm/h transdermales Pflaster (Grünenthal $\mathrm{GmbH}$ ), as well as the comedication used in the study for opioid-receptors blockade (Naltrexon$\mathrm{HCl}$ neuraxpharm ${ }^{\circledast} 50 \mathrm{mg}$ Filmtabletten of Neuraxpharm Arzneimittel $\mathrm{GmbH}$ ) were sourced from the German market. The Test product, Buprenorphin TDS $70 \mu \mathrm{g} / \mathrm{h}$ transdermal patch, was manufactured by AMW GmbH in a GMP production facility located in Germany.

\section{Adhesion performance study in healthy human volunteers}

Study design and participants: An open label, randomized, single dose, two-period, two-sequence, crossover adhesion performance study was conducted in healthy male and female volunteers. During each period, either a Test or a Reference patch was administered, according to the randomization table. Both formulations were transdermal drug delivery systems containing buprenorphine, with a nominal release rate of 70 micrograms per hour. The application site selected for the study was the upper chest, bellow the collar bone, in accordance with the Patient Information Leaflet of the Reference product recommending an "area of skin which is flat, clean and hairless, located on the upper body, preferably on the chest below the collar bone". The same application area located on the left side of the chest was used in both study periods in order to ensure that vascularization of the site and muscle movement patterns have no impact on the MIT results in the present cross-over setting. Each patch was worn in standardized conditions for 96 consecutive hours (4 days). A naltrexone blockade was used in the study in order to reduce the risk for opioid-related adverse events. The actual length of the washout period was of 16 days between patch removal on Period I and patch application on Period II, in accordance to a minimum requirement of 14 days before reusing the same application site (measure of precaution taken for safety reasons related to local tolerability).

The study was conducted following unconditional approval from the National Ethics Committee, the Ethics Committee of the Clinical Hospital of the Ministry of Health of the Republic of Moldova and the Medicine Agency. Clinical investigations were conducted according to the Declaration of Helsinki principles and Good Clinical Practice.

All subject gave their written informed consent before they underwent any study-related procedures and were free to withdraw from the trial at any time. The demographic characteristics of the subject participating in the study are presented in Table 1.

\begin{tabular}{|c|c|}
\hline $\begin{array}{c}\text { Characteristic (method of data } \\
\text { presentation) }\end{array}$ & In vivo adhesion performance study \\
\hline Number of subject & $\begin{array}{c}\text { Enrolled: } 26 \text { Dosed at least once: } \\
\text { 26 Completed the study: } 24\end{array}$ \\
\hline $\begin{array}{c}\text { Gender distribution for dosed subject } \\
\text { (number, } \% \text { of study population)) }\end{array}$ & $\begin{array}{c}\text { Female: } 13(50.00 \%) \\
\text { Male: } 13(50.00 \%)\end{array}$ \\
\hline $\begin{array}{c}\text { Race of dosed subject } \\
\text { (number, }(\% \text { of study population)) }\end{array}$ & Caucasian: $26(100.00 \%)$ \\
\hline $\begin{array}{c}\text { Age of dosed subject } \\
\text { (mean, }( \pm \text { standard deviation)) }\end{array}$ & $28.04( \pm 7.82)$ \\
\hline $\begin{array}{c}\text { Body mass index of dosed subject } \\
\text { (mean, ( } \pm \text { standard deviation)) }\end{array}$ & $23.63( \pm 2.94)$ \\
\hline
\end{tabular}

Table 1: Demographic characteristics of the population included in the in vivo adhesion performance study.

Collection and analysis of adhesion data: Per Protocol assessment of adhesion performance was based on individual observations 
Citation: Duna SN, Ghita A, Grasser S, Rizea- Savu S (2018) Innovative Use of Medical Infrared Thermography to Evaluate Adhesive Properties of Two Buprenorphine Transdermal Patch Formulations in a Single Dose Adhesion Performance Study. J Bioequiv Availab 10: 92-98. 384. doi: 10.4172/0975-0851.1000384

regarding the percentage of patch area that remained adhered at $0.5,12,24,36,48,60,72,84$ and 96.00 hours post application. Grids of the same shape and area like the Test and Reference patches were developed, printed onto transparent sheets (Xerox Premium Transparencies) and used for the acquisition of adhesion data through human visual observation. Areas of the patch where lift-off occurred were drawn on the pattern with precision black permanent markers. While the drawing was made, the grids were kept in close vicinity to the patch, directly above it, but the assessor made sure to avoid contact between the two. For standardization, the marking of lifted areas on grids was performed only by one trained staff member for all adhesion performance evaluations carried out during the study. The marked grids were then scanned and used to determine the overall area that exhibits detachment by using ImageJ software for the quantification of the sum of areas marked in black. ImageJ is a software for the analysis of scientific images developed by the US National Institute of Health [8], validated in-house for its use in adhesion performance studies.

Photographs of the transdermal patches were taken at each evaluation time point as supportive documentation of adhesion status (taken with a Lumix DMC-G6 camera (Panasonic, Japan) in standardized conditions).

Also, thermal images were acquired during each adhesion performance evaluation, using a Meditherm med2000 system for biomedical applications. Data capture was carried out using WinTES2 software (version 1.00.0030, Compix Inc., USA 2009) in Medical.256.map display mode, as appropriate for medical applications involving a physiological temperature range. An acquisition range of $8^{\circ} \mathrm{C}$ was used in order to obtain best contrast for detached areas while still accounting for inter- individual variations relating to skin surface temperature. The thermal resolution was $0.1^{\circ} \mathrm{C}$ and each pixel of the thermogram was an individually referenced temperature reading. Ambient temperature conditions, direction and duration of forced airflow used for temperature gradient enhancement between attached and detached areas as well as camera to patch distance were standardized. Furthermore, any distance variability that may have still resulted (due to slightly distinct posture or chest curvature) was accounted for during image analysis, pixels to millimeters ratio being adjusted for actual patch size. In order to quantify detachment percentages from thermograms, the same in-house validated version of the ImageJ software (version 1.47v; Java 1.6.0_20 [32 bit], National Institute of Health, USA) was again used. The software was operated in Windows 8.1 (Microsoft Corp., USA). At the time of study conduct, adhesion results were presented as per study protocol, based on the evaluation of grid markings. The presented analysis of thermograms was performed post-hoc, as part of the third validation step of the buprenorphine-specific adhesion assessment through thermal imaging methodology, which involves the comparison of results obtained by manual versus thermographic assessments. Method suitability tests have been conducted prior to the analysis in order to check image resolution and surface cooling effect within the physiological temperature range, reproducibility and accuracy over time (in vitro experiments with actual study patches applied on warmed plastic blocks maintained at constant physiological temperature, which contained drilled orifices of known width and depth simulating reallife detachment scenarios). Based on positive and satisfactory results of the first validation phase, method reproducibility, accuracy and specificity were then tested on human subject wearing Test placebo patches (courtesy of AMW GmbH, Germany) in conditions designed to create controlled and reproducible detachment areas of known size.

\section{Statistical analysis of adhesion data}

Mean adherence of the study formulations (Test and Reference) at the end of the dosing interval ( $96 \mathrm{~h}$ post-application) as well as the associated $90 \%$ confidence intervals were determined using the GLM procedure in SAS statistical software, version 9.4 (SAS Institute Inc., USA). As per applicable European Guidelines [2], since the $90 \%$ confidence interval of mean adherence for the Test product did not lie above $90 \%$ and the same was found also for the Reference product, the primary criteria for adhesion performance evaluation became the assessment of non-inferiority. Therefore, the $90 \%$ confidence interval for the difference of adhesiveness (Test - Reference) at the end of the dosing interval, using the percentage of adhesion as continuous variable, was determined using the GLM procedure in SAS. A lower limit of the $90 \%$ confidence interval for the difference of adhesiveness not lower than $-10 \%$ was required for demonstration of non-inferiority of the Test product.

\section{Results and Discussion}

The working hypothesis for this thermography adhesion assessment project was that patch surface temperature mapping can detect real discontinuity from skin with more sensitivity than visual examination. Validation tests for the thermographic approach had already revealed some of the major advantages of this method: day-to-day and subsequent assessment reproducibility, accuracy to detect known areas of detachment, sensitivity to correctly detect detachment areas of $1 \mathrm{~mm}$ width and $1 \mathrm{~mm}$ depth (possibly even higher considering the resolution of the used thermo camera, although a lower threshold could not be tested during in-vitro tests due to drilling hardware limitations) and high- throughput characteristics (one evaluation taking 30 seconds, cooling phase included). However, it was not known how the thermographic assessment would compare against the standard visual evaluation in a clinical setting, during a real study. A post-hoc analysis of adhesion was therefore conducted on thermographs acquired at the end of the dosing interval (time point considered for noninferiority assessment as per European Guidelines) during an adhesion performance study conducted on two buprenorphine formulations with a 96-hours labeled duration of wear and 70 micrograms per hour nominal release rate. The results obtained via MIT were compared against reported study results, which relied on analysis of marked grid patterns following human visual examination. The statistical results presented in this article are representative for the Per Protocol Study Population, comprised of 24 out of the 26 subject enrolled, which completed the study and provided evaluable adhesion data for both formulations. Two subject discontinued the study prematurely, due to reasons unrelated to adhesion performance (one subject was withdrawn at the Investigator's decision because the patch applied in the first study period was not well-tolerated locally, while another subject dropped out due to personal reasons).

Mean results were comparable among the two methods (Table 2), and the conclusion of noninferiority of the Test product was confirmed also through analysis of thermograms (the lower limit of the $90 \%$ confidence interval for Mean Adhesion Difference being not lower than $-10 \%)$.

When compared to the visual examination, the analysis of infrared thermograms provided slightly higher mean adhesion results for both formulations. This was to be expected considering the fact that assessors are trained to always use the "worst-case scenario" approach when marking the areas of detachment in order to avoid unwilling bias. Therefore, in addition to clear detachment situations, the following are 
Citation: Duna SN, Ghita A, Grasser S, Rizea- Savu S (2018) Innovative Use of Medical Infrared Thermography to Evaluate Adhesive Properties of Two Buprenorphine Transdermal Patch Formulations in a Single Dose Adhesion Performance Study. J Bioequiv Availab 10: 92-98. 384. doi: 10.4172/0975-0851.1000384

also marked on grids: irregularities noted on the median section of the patch which look like air bubbles possibly causing discontinuity between patch and skin; significant wrinkling which seems accompanied by lift-off from the skin; deteriorations of the visible surface of the patch

\begin{tabular}{|c|c|c|c|}
\hline $\begin{array}{l}\text { Source Data } \\
\text { Evaluated }\end{array}$ & Formulation & $\begin{array}{c}\text { Adhesion } \\
\text { Percentage at } \\
\text { 96.00h LSMEAN } \\
(90 \% \mathrm{Cl})\end{array}$ & $\begin{array}{c}\text { Mean Adhesion Difference } \\
\text { TEST - REFERENCE ( } 90 \% \\
\text { CI) }\end{array}$ \\
\hline \multirow{2}{*}{ Marked Grids } & Test & $\begin{array}{c}88.205(85.842- \\
90.568)\end{array}$ & \multirow[b]{2}{*}{$4.119(0.899-7.338)$} \\
\hline & Reference & $\begin{array}{c}84.086(81.906- \\
86.267)\end{array}$ & \\
\hline \multirow{2}{*}{$\begin{array}{c}\text { Infrared } \\
\text { Thermo grams }\end{array}$} & Test & $\begin{array}{c}90.462(87.812- \\
93.112)\end{array}$ & \multirow[b]{2}{*}{$4.874(1.264-8.484)$} \\
\hline & Reference & $\begin{array}{c}85.589(83.144- \\
88.034)\end{array}$ & \\
\hline
\end{tabular}

Table 2: Comparison of study results obtained through analysis of grid markings vs. MIT. (tearing at the edges of the textile over tape after a prolonged period of wear).

Individual results obtained for the Test formulation ( $\mathrm{n}=24$ subject) were mostly similar among the two evaluation methods (see graphical representation in Figure 1), data from 20 subject differing by no more than $\pm 5 \%$, data from 3 subject differing by no more than $\pm 8 \%$ and one outlying observation where the difference exceeded $10 \%$, which will be subsequently discussed as Case Study 1.

In the case of subject 26 , adhesion results differed by more than $10 \%$ when comparing between assessment of marked grid $(81.36 \%$ adhesion percentage) and thermogram (93.13\% adhesion percentage). Patch surface irregularities (apparent lift-off) were noted at the visual inspection of the central patch area and marked as detachment on the evaluation grid; meanwhile, MIT revealed only minor discontinuity between patch and skin on the respective area. In taking a close look at the photographs taken immediately after patch removal, edema of the

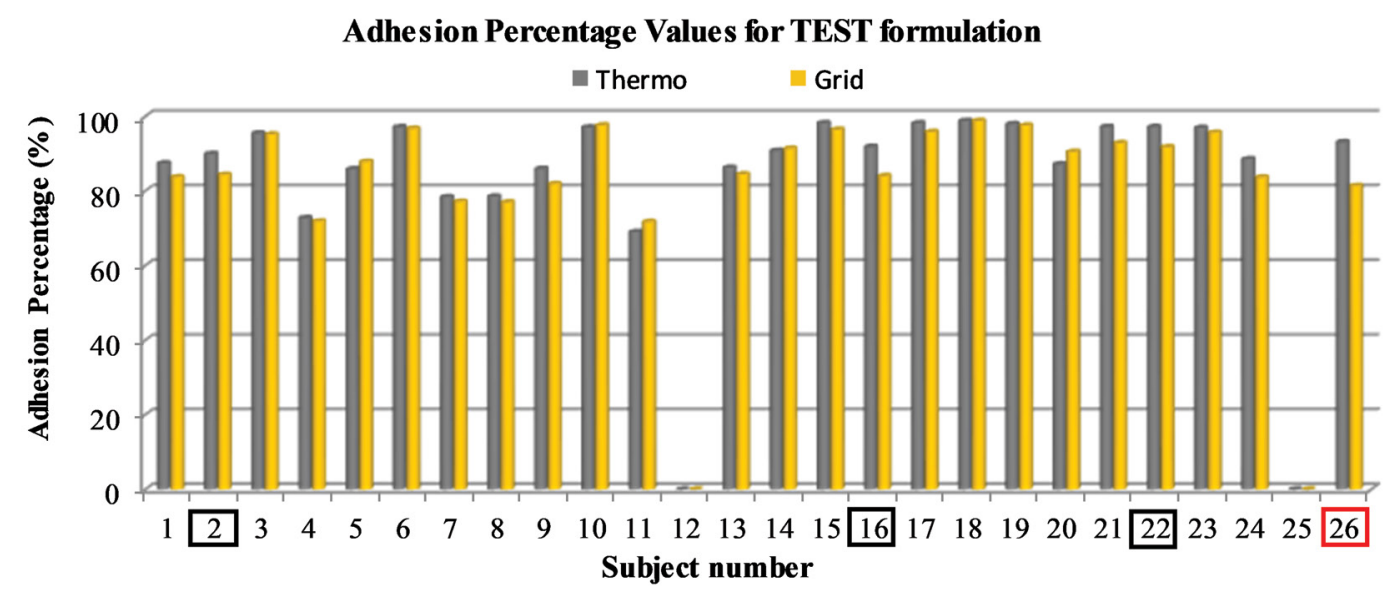

Figure 1: Presentation of adhesion percentages quantified for the Test formulation by MIT vs. grid markings.

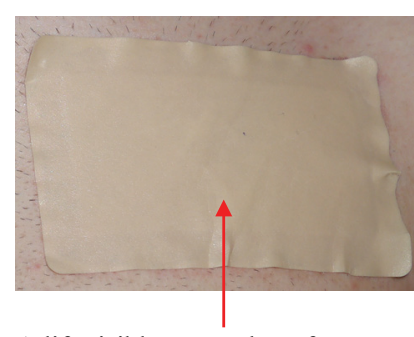

A lift visible on patch surface

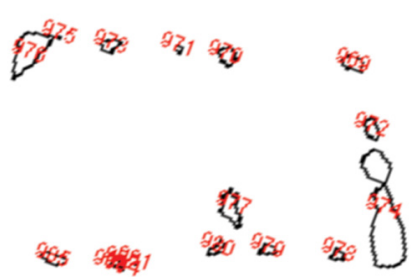

MIT particle analysis

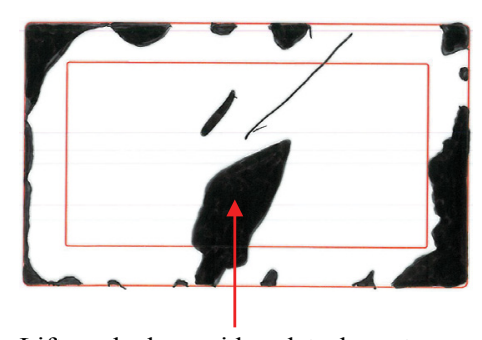

Lift marked on grid as detachment

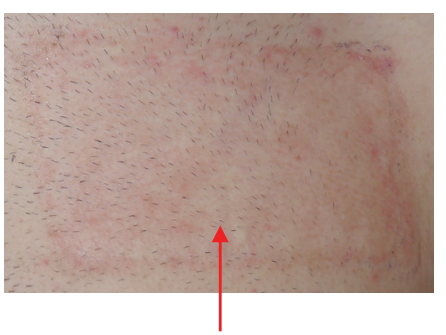

Post-removal photo shows skin edema

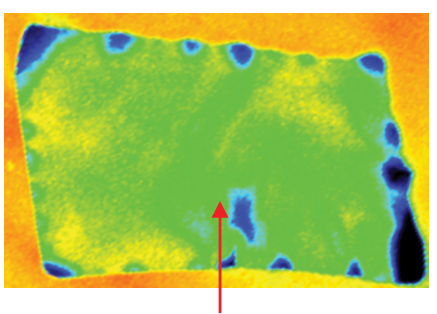

MIT reveals only minor detachment

Figure 2: Case Study 1 - Overestimation of detachment percentage at visual examination due to edema of the underlying skin. 
Citation: Duna SN, Ghita A, Grasser S, Rizea- Savu S (2018) Innovative Use of Medical Infrared Thermography to Evaluate Adhesive Properties of Two Buprenorphine Transdermal Patch Formulations in a Single Dose Adhesion Performance Study. J Bioequiv Availab 10: 92-98. 384. doi: 10.4172/0975-0851.1000384

\section{Adhesion Percentage Values for REFERENCE formulation}

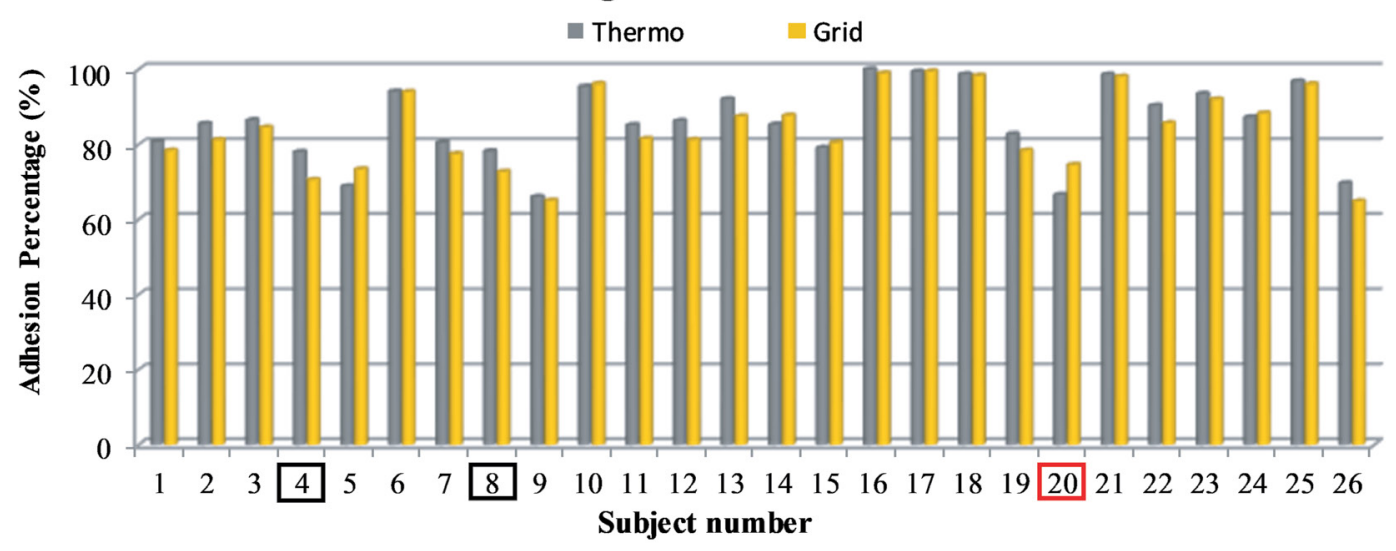

Figure 3: Presentation of adhesion percentages quantified for the Reference formulation by MIT vs. grid markings.
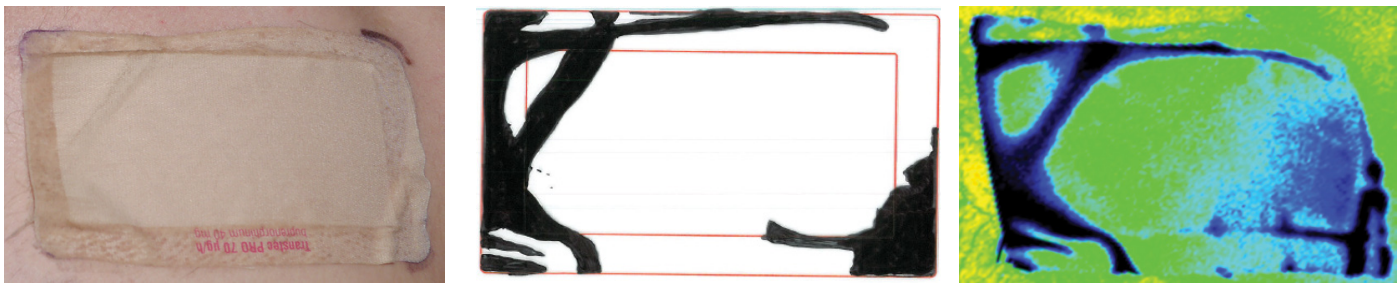

Figure 4: Case Study 2 - MIT reveals detachment of core area not readily visible on patch surface.
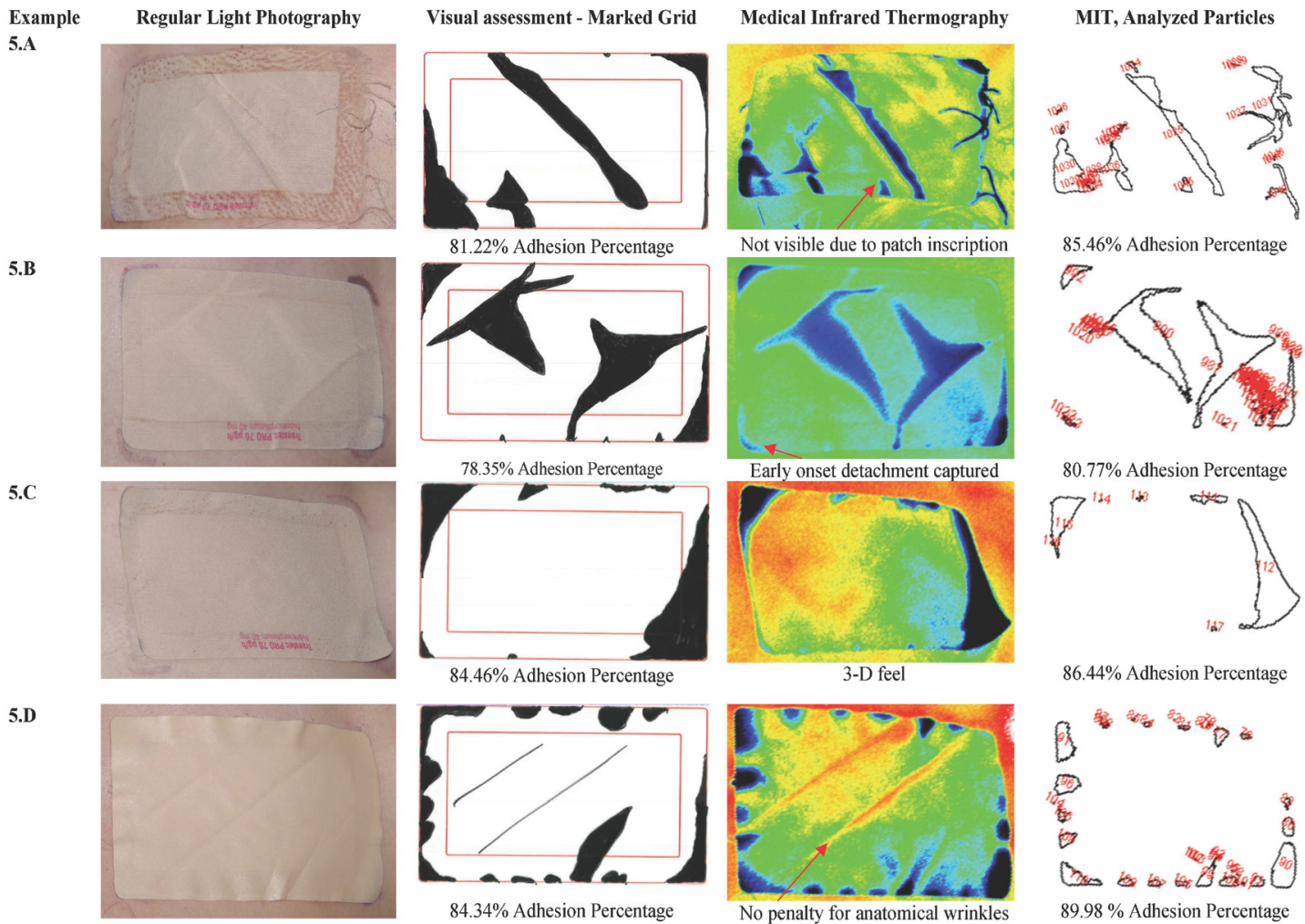

Observation

MIT- better at

depicting tear-

off situations

and detecting

detachment

areas masked

by inscriptions

$81.22 \%$ Adhesion Percentage

Not visible due to patch inscription

$85.46 \%$ Adhesion Percentage

discoloring
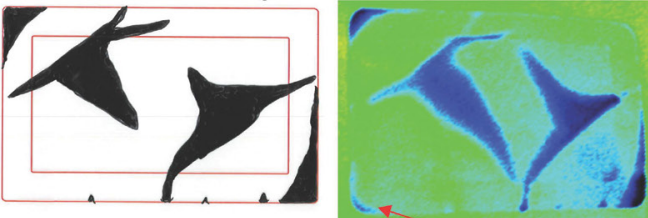

$\sqrt{2}$

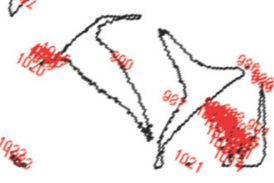

MIT helps

identify also

early onset

detachment,

not

discernable

through visual
examination

$80.77 \%$ Adhesion Percentage
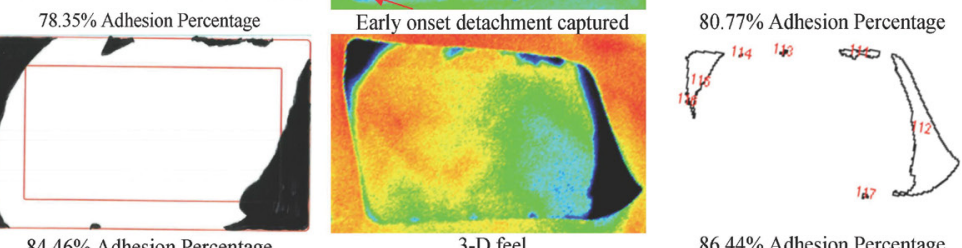

MIT provides

a 3D feel that

highlights

major lift-off,

correctly

quantified on

quantified on

$84.46 \%$ Adhesion Percentage

86.44\% Adhesion Percentage

but not

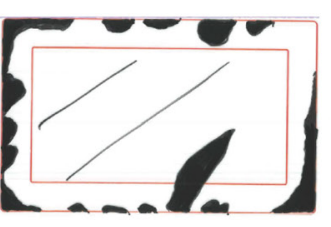

84.34\% Adhesion Percentage

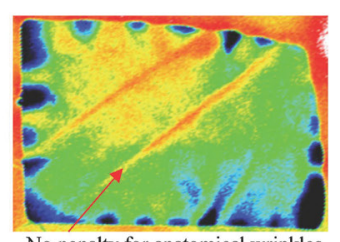

No penalty for anatomical wrinkles
等

89.98 \% Adhesion Percentage
MIT is

sensitive

enough to

anatomic

wrinkles are

coupled with

coupled with

not

Figure 5: Representative examples of patch detachment situation, side by side comparison of results obtained through visual examination vs. MIT. 
Citation: Duna SN, Ghita A, Grasser S, Rizea- Savu S (2018) Innovative Use of Medical Infrared Thermography to Evaluate Adhesive Properties of Two Buprenorphine Transdermal Patch Formulations in a Single Dose Adhesion Performance Study. J Bioequiv Availab 10: 92-98. 384. doi: 10.4172/0975-0851.1000384

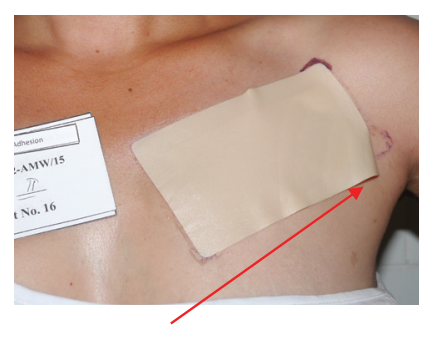

Patch corner bent inwards

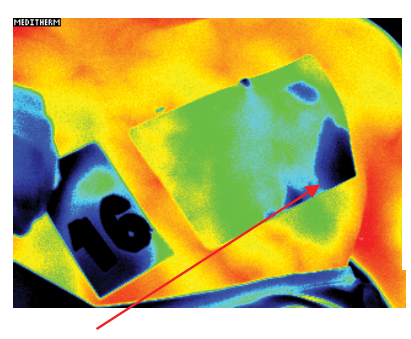

Corner area not quantified through MIT
Tfore

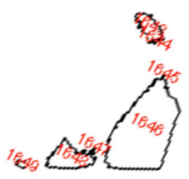

MIT analysis: $91.87 \%$ adhesion

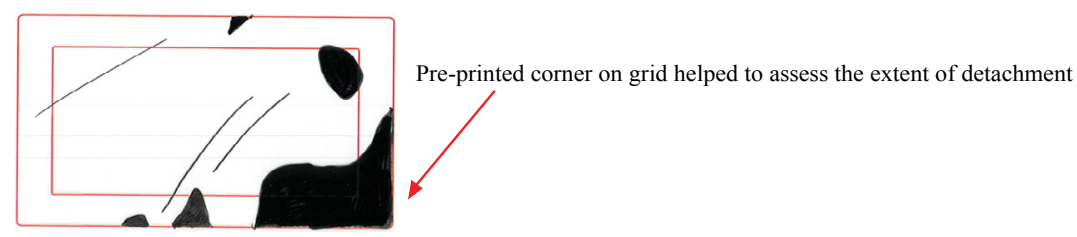

Grid analysis: $84.01 \%$ adhesion

Figure 6: Overestimation of adhesion percentage by MIT due to patch bending.

skin area beneath that part of the patch was observed, thus explaining why the patch seemed lifted at visual inspection but was yet in contact with the skin as shown by the thermogram (Figure 2).

For the Reference formulation ( $\mathrm{n}=26$ subject), individual results were also mostly similar among the two evaluation methods (see graphical representation of individual results for Reference in Figure 3 ), data from 23 subject differing by no more than $\pm 5 \%$ and data from 3 subject differing by no more than $\pm 8 \%$; the largest difference noted (7.94\%) will be subsequently discussed as Case Study 2.

In the case of subject 20 , adhesion results differed by almost $8 \%$ when comparing between assessment of marked grid $(74.52 \%$ adhesion percentage) and thermogram (66.58\% adhesion percentage) because MIT revealed early onset detachment of core area not readily visible on the overlay surface (Figure 4).

Apart from the comparison of mean and individual adhesion results obtained for all subject, each individual thermogram was visually compared against the grid marked and the regular photograph taken at the same evaluation time point, in order to identify possible advantages (Figure 5) and limitations of MIT as adhesion assessment tool.

The following advantages of infrared thermography were noted during critical analysis of the data: it is better at depicting tear-off situations and at detecting detachment areas that can be overlooked at visual examination because they are masked by discoloring of the patch overlay (occurs when patches formulated with textile overlay are worn for a prolonged period of time by subject who sweat abundantly) and/or by inscriptions (Figure 5A); it helps identify also early onset detachment, not discernable through visual examination (Figures $5 \mathrm{~B}$ and 4); it provides a three dimensional feel that highlights major lift-off (Figure 5C); it is sensitive enough to detect whether anatomic wrinkles are coupled with detachment or not (Figure 5D) and to discriminate between lift-off caused by air gaps and lift-off caused by edema of the skin beneath the patch (Figure 2).

One disadvantage of MIT was also noted, relating to the seldom encountered situation when a patch area lifting-off bends inwards thus causing an overestimation of adhesion percentage due to the fact that part of the detached area is not visible on the thermogram (Figure 6).
A standardized methodology for handling such situations must be developed and transparently applied when using MIT as adhesion assessment tool. Among the options that can be considered are: switching to visual examination as primary when such a case is encountered and corner reconstruction prior to particle analysis, based on the known size and shape of the TDS. Any of the proposed manual interventions would constitute a protocol deviation and transparency would be ensured through adequate justification and description of the manual intervention process and its impact on results.

\section{Conclusion}

Data review has provided very compelling evidence that Medical Infrared Thermography (MIT) is far superior to normal light photography as imagistic tool for documenting patch detachment in clinical studies.

In terms of quantification of detachment, MIT presents a much lower risk, as compared to the classical manual marking on grid, of bias or unwilling data alteration during collection (no risk of accidentally pressing the grid against the patch while performing an evaluation or of overlooking to mark one of the tinier areas detached etc.). Furthermore, the process can be standardized up to the point where the human factor has negligible impact on the acquired data, thus solving the problem of between-assessors and within-assessor variability. MIT was also shown to be more selective in detecting real detachment as compared to visual examination.

The reproducibility, accuracy, sensitivity and acquisition speed of MIT are all attributes that recommend it as an assessment method more consistent with current regulatory requirements for standardization of clinical trials as compared to human visual examination. The critical analysis of individual data has served its purpose of identifying the numerous advantages and the limitation which have to be studied more in-depth before a robust product-specific method validation can be completed.

Convinced that MIT represents the future of patch adhesion evaluation, our work will continue with analysis of thermography 
Citation: Duna SN, Ghita A, Grasser S, Rizea- Savu S (2018) Innovative Use of Medical Infrared Thermography to Evaluate Adhesive Properties of Two Buprenorphine Transdermal Patch Formulations in a Single Dose Adhesion Performance Study. J Bioequiv Availab 10: 92-98. 384. doi: 10.4172/0975-0851.1000384

data from several other buprenorphine studies performed in-house, until satisfied that the wide range of detachment scenarios possibly encountered in the clinic can be captured and assessed through thermography directly or through clear contingency plans. Though equipment acquisition costs are high and proper method validation has turned out to be a lengthy and complex process, the accuracy, sensitivity and level of standardization achieved justified the efforts. In the opinion of the authors, the benefits of this method far outweigh the one easy-to-overcome limitation identified, making MIT an ideal tool for assessment of adhesion performance both in the early screening phase of pilot batches and in a pivotal setting where comparisons are carried out against a Reference market standard.

\section{References}

1. http://www.agah.eu/uploads/tx_news/Schriever_patch_adhesion_local_ tolerability.pdf

2. EMA - CHMP (2014) Guideline on the pharmacokinetic and clinical evaluation of modified release dosage forms (EMA/CPMP/EWP/280/96 Corr1)
3. FDA - CDER (2016) Assessing Adhesion with Transdermal Delivery Systems and Topical Patches for ANDAs

4. Hildebrandt C, Raschner C, Ammer K (2010) An Overview of Recent Application of Medical Infrared Thermography in Sports Medicine in Austria. Sensors 10 : 4700-4715.

5. Avdelidis NP, Gan TH, Ibarra-Castanedo C, Maldague XPV (2011) Infrared thermography as a non-destructive tool for materials characterisation and assessment. Proceedings of the Thermosense: Thermal Infrared Applications XXXIII.

6. Szentkuti A, Kavanagh HS, Grazio S (2011) Infrared thermography and image analysis for biomedical use. Periodicum Biologorum 113: 385-392.

7. https://www.engineersedge.com/heat_transfer/convective_heat_transfer_ coefficients13378.htm

8. Schneider CA, Rasband WS, Eliceiri KW (2012) NIH Image to ImageJ: 25 years of image analysis. Nature Methods 9: 671-675 